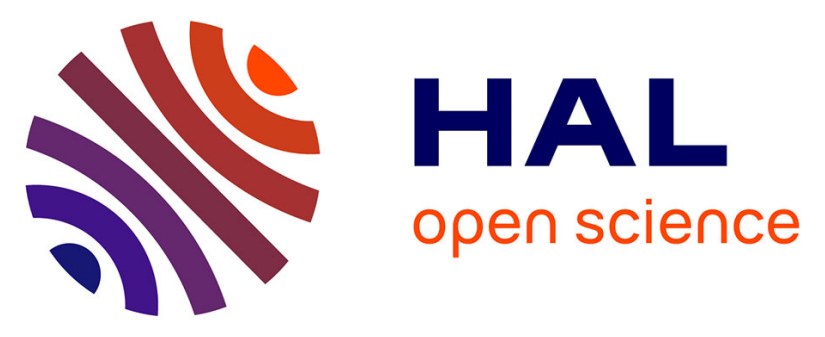

\title{
Co-immobilization of a Rh Catalyst and a Keggin Polyoxometalate in the UiO-67 Zr-Based Metal-Organic Framework: In Depth Structural Characterization and Photocatalytic Properties for CO2 Reduction
}

Youven Benseghir, Alex Lemarchand, Mathis Duguet, Pierre Mialane, Maria Gomez-Mingot, Catherine Roch-Marchal, Thomas Pino, Minh-Huong Ha-Thi, Mohamed Haouas, Marc Fontecave, et al.

\section{- To cite this version:}

Youven Benseghir, Alex Lemarchand, Mathis Duguet, Pierre Mialane, Maria Gomez-Mingot, et al.. Co-immobilization of a Rh Catalyst and a Keggin Polyoxometalate in the UiO-67 Zr-Based Metal-Organic Framework: In Depth Structural Characterization and Photocatalytic Properties for CO2 Reduction. Journal of the American Chemical Society, 2020, 142 (20), pp.9428-9438. 10.1021/jacs.0c02425 . hal-02878697

\section{HAL Id: hal-02878697 \\ https: / hal.sorbonne-universite.fr/hal-02878697}

Submitted on 29 Jun 2020

HAL is a multi-disciplinary open access archive for the deposit and dissemination of scientific research documents, whether they are published or not. The documents may come from teaching and research institutions in France or abroad, or from public or private research centers.
L'archive ouverte pluridisciplinaire $\mathbf{H A L}$, est destinée au dépôt et à la diffusion de documents scientifiques de niveau recherche, publiés ou non, émanant des établissements d'enseignement et de recherche français ou étrangers, des laboratoires publics ou privés. 


\title{
Co-immobilization of a Rh catalyst and a Keggin Polyoxometalate in the UiO-67 Zr-based Metal-Organic- Framework: in Depth Structural Characterization and Photocatalytic Properties for $\mathrm{CO}_{2}$ Reduction.
}

\author{
Youven Benseghir, ${ }^{\S, \neq}, \|$ Alex Lemarchand, ${ }^{\S, \|}$ Mathis Duguet, ${ }^{\S, \neq}$ Pierre Mialane, , Maria Gomez- \\ Mingot, ${ }^{\S}$ Catherine Roch-Marchal, $\neq$ Thomas Pino, ${ }^{\perp}$ Minh-Huong Ha-Thi, ${ }^{\perp}$ Mohamed Haouas, ${ }^{\ddagger}$ Marc \\ Fontecave, ${ }^{\S}$ Anne Dolbecq, ${ }^{*}$, Capucine Sassoye, ${ }^{*}, \dagger$ and Caroline Mellot-Draznieks ${ }^{*}, \$$
}

$\S$ Laboratoire de Chimie des Processus Biologiques, UMR CNRS 8229, Collège de France, Sorbonne Université, PSL Research University, 11 Place Marcelin Berthelot, 75231 Paris Cedex 05, France

‡ Université Paris-Saclay, UMR CNRS 8180, Université de Versailles Saint-Quentin en Yvelines, Institut Lavoisier de Versailles, 45 Avenue des Etats-Unis, 78000 Versailles, France

† Sorbonne Université, UMR 7574, Collège de France, Laboratoire de Chimie de la Matière Condensée de Paris, 4 Place Jussieu, 75252 Paris cedex 05, France

${ }^{\perp}$ Université Paris-Saclay, CNRS, Institut des Sciences Moléculaires d'Orsay, 91405, Orsay, France

\begin{abstract}
The Keggin-type polyoxometalate (POM) $\mathrm{PW}_{12} \mathrm{O}_{40^{3-}}$ and the catalytic complex $\mathrm{Cp}^{*} \mathrm{Rh}\left(\right.$ bpydc) $\mathrm{Cl}_{2}$ (bpydc $=2,2^{\prime}$ bipyridine-5,5'-dicarboxylic acid) were co-immobilized in the $\operatorname{Zr}(\mathrm{IV})$ based metal organic framework UiO-67. The POM is encapsulated within the cavities of the MOF by in situ synthesis and then, the Rh catalytic complex is introduced by postsynthetic linker exchange. Infrared and Raman spectroscopies, ${ }^{31} \mathrm{P}$ and ${ }^{13} \mathrm{C}$ MAS NMR, $\mathrm{N}_{2}$ adsorption isotherms and X-ray diffraction indicate the structural integrity of all components (POM, Rh-complex and MOF) within the composite of interest (PW12,Cp*Rh)@UiO-67. DFT calculations identified two possible locations of the POM in the octahedral cavities of the MOF: one at the center of a UiO-67 pore with the $\mathrm{Cp} * \mathrm{Rh}$ complex pointing towards an empty pore and one off-centered with the $\mathrm{Cp}{ }^{*} \mathrm{Rh}$ pointing towards the POM. ${ }^{31} \mathrm{P}-{ }^{1} \mathrm{H}$ heteronuclear (HETCOR) experiments ascertained the two environments of the POM, equally distributed, with the POM in interaction either with the $\mathrm{Cp}^{*}$ fragment or with the organic linker. In addition, Pair Distribution function (PDF) were collected on the POM@MOF composite and provided key evidence of the structural integrity of the POM once immobilized into the MOF. The photocatalytic activity of the (PW $\left.\mathrm{PW}_{12}, \mathrm{Cp} * \mathrm{Rh}\right) @ U i 0-67$ composite for $\mathrm{CO}_{2}$ reduction into formate and hydrogen were evaluated. The formate production was doubled when compared with that observed with the POM-free Cp*Rh@UiO-67 catalyst and reached TONs as high as 175 when prepared as thin films, showing the beneficial influence of the POM. Finally, the stability of the composite was assessed by means of recyclability tests. The combination of XRD, IR, ICP and PDF experiments were essential in confirming the integrity of the POM, the catalyst and the MOF after catalysis.
\end{abstract}

\section{INTRODUCTION}

In the current energetic transition, the photoreduction of $\mathrm{CO}_{2}$ to energy-dense organic molecules $\left(\mathrm{CO}, \mathrm{HCOOH}, \mathrm{CH}_{2} \mathrm{O}, \mathrm{CH}_{3} \mathrm{OH}\right.$, etc.) under sunlight is considered as a green and sustainable however challenging strategy. The heterogenization of molecular catalysts (Cats) is an attractive strategy which combines the advantages of homogeneous catalysis (high activity, selectivity, tuning of the catalytic activity via structural modification) and those of heterogeneous catalysis (easy recovery, thin films processability). ${ }^{1}$ In this respect, Metal-Organic-Frameworks (MOFs) attract an ever-growing interest as platforms to develop synthetic or post-synthetic strategies for the immobilization of catalysts composites for photo- and electrocatalysis purposes. ${ }^{2}$ In the so-called Cat@MOF, the confinement of the catalyst in the MOF's pores is expected to increase its stability while modifying its selectivity. Only few active Cat@MOF photosystems ${ }^{3}$ have been reported so far in the field of photocatalytic $\mathrm{CO}_{2}$ reduction, focusing mainly on UiO-67 Zr-MOF functionalized with $\mathrm{Re}^{4,5} \mathrm{Ru}^{6,7} \mathrm{Rh}^{7,8}$ or $\mathrm{Mn}^{9}$ catalytic complexes as constitutive linkers, UiO-66 MOF with $\mathrm{Cr}$-monocatecholato ligands ${ }^{10}$ or the very large pore MIL-101- $\mathrm{NH}_{2}$ with a molecular $\mathrm{Cp} * \mathrm{Rh}$ catalyst encapsulated in the cavities of the MOF.11

In our earlier work, we provided evidence for the photosensitization of the $\mathrm{Cp} * \mathrm{Rh}$ bipyridine complex in the $\mathrm{Cp} * \mathrm{Rh} @ U \mathrm{UiO}-67$ composite for $\mathrm{CO}_{2}$ reduction under visible light illumination. ${ }^{8}$ In another context, we successfully encapsulated for the first time the Keggin-type $\mathrm{PW}_{12} \mathrm{O}_{40^{3-}}\left(\mathrm{PW}_{12}\right)$ polyoxometalate (POM) in UiO-67 through in situ synthesis of the POM@MOF.12 
POMs are soluble anionic metal oxide clusters of d-block transition metals in high oxidation states (typically $\mathrm{W}^{\mathrm{VI}}$, Mo $\mathrm{V}^{\mathrm{V}, \mathrm{VI}}$, or $\mathrm{V}^{\mathrm{IV}, \mathrm{V}}$ ). They exhibit remarkable redox and acid-base properties and have proved to be efficient catalysts for various reactions $^{13}$ such as oxidation and acid catalysis of organic substrates, oxygen and hydrogen evolution ${ }^{14}$ reactions
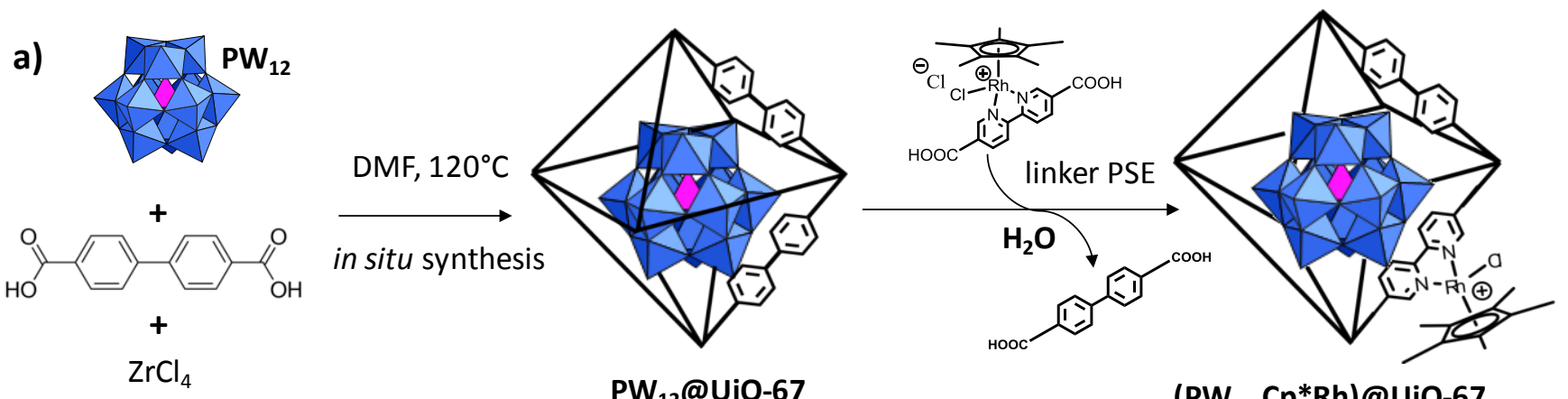

PW $12 @ U i 0-67$

$\left(\mathrm{PW}_{12}, \mathrm{Cp} * \mathrm{Rh}\right) @ U i 0-67$
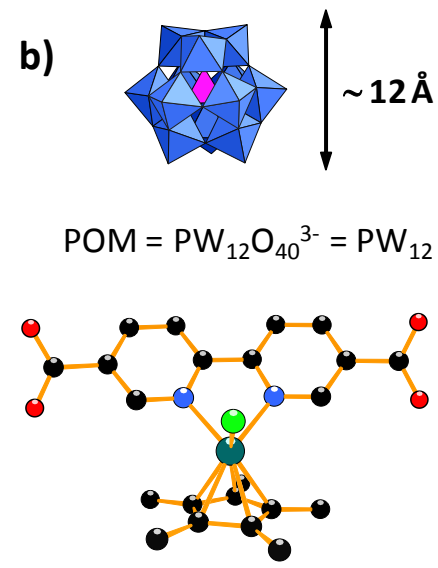

catalyst $=[\mathrm{Cp} * \mathrm{Rh}(\mathrm{bpydc}) \mathrm{Cl}]^{+}$
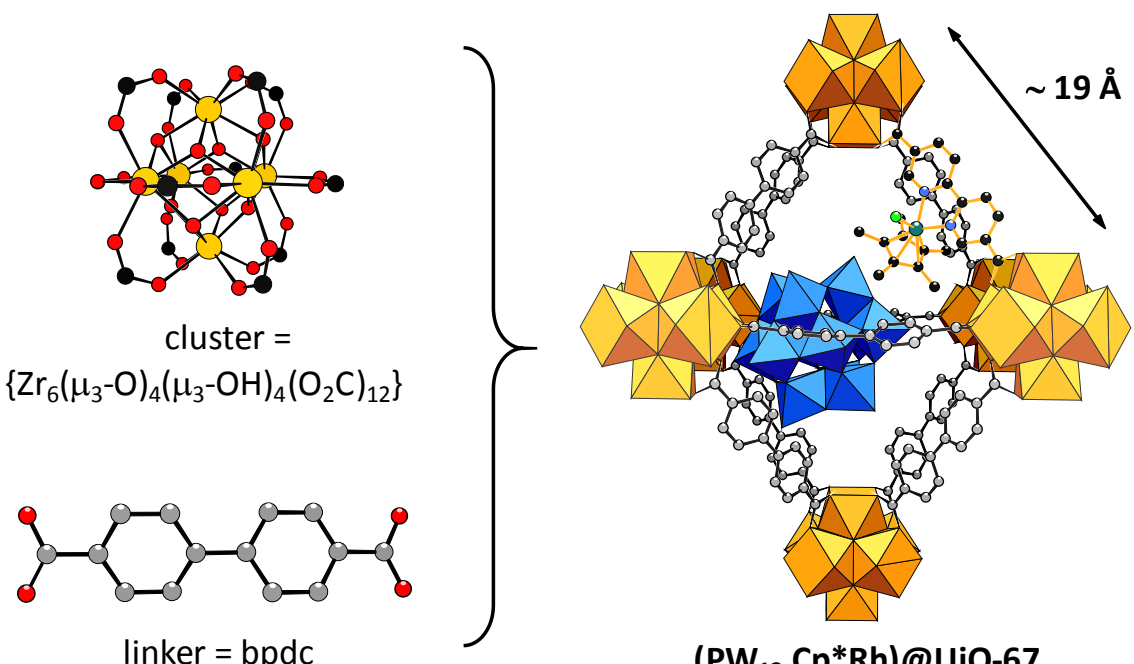

$\left(P W_{12}, C p * R h\right) @ U i O-67$

Figure 1. (a) Schematic representation of the two-step in situ synthesis and linker's post-synthetic exchange (PSE) procedure to obtain the composite material (PW $12, \mathrm{Cp} * \mathrm{Rh}) @ U i 0-67$. (b) (PW $\left.12, \mathrm{Cp}^{*} \mathrm{Rh}\right) @ U i 0-67$ 's components: the PW12 polyoxometalate, the Zrbased inorganic sub-unit of UiO-67, the $[\mathrm{Cp} * \mathrm{Rh}(\mathrm{bpydc}) \mathrm{Cl}]^{+}$molecular catalyst and the bpdc linkers. The position of the POM is obtained from density function theory (DFT) calculations (see text). $\mathrm{WO}_{6}$, blue polyhedra; $\mathrm{ZrO}_{8}$, orange polyhedra; $\mathrm{PO}_{4}$, pink polyhedron; $\mathrm{Zr}$, orange spheres; $\mathrm{O}$, red spheres; $\mathrm{C}$, gray or black spheres; $\mathrm{N}$, blue spheres, $\mathrm{Rh}$, dark green spheres; $\mathrm{Cl}$, green spheres.

with however very scarce examples for $\mathrm{CO}_{2}$ conversion. ${ }^{15}$ We can cite a few remarkable examples of materials containing $\left\{\mathrm{P}_{4} \mathrm{MoV}_{6}\right\}$ units ${ }^{16}$ or $\left\{\varepsilon-\mathrm{PMo}_{8} \mathrm{Mo}^{\mathrm{VI}} \mathrm{Zn}_{4}\right\}$ Keggin ions linked to hydrophobic ${ }^{17}$ or porphyrinic ${ }^{18}$ linkers. Furthermore, due to their ability to store electrons/protons, POMs can serve as electrons/protons relays in association with catalytic species. For example, hybrid compounds with $\mathrm{Re}^{\mathrm{I}}$ complexes associated to the Keggin-type $\mathrm{PW}_{12}$ were prepared and a reaction pathway was proposed for the photoreduction of $\mathrm{CO}_{2}$ to $\mathrm{CO}$ involving the reduced and protonated POM. ${ }^{19-21}$ In another example $\mathrm{PW}_{12}$ at the surface of $\mathrm{Pt}$ nanoparticles was reported to be able to transfer electrons from the photoexcited amino ligand of $\mathrm{NH}_{2}$-MIL-53 to the Pt catalyst where $\mathrm{H}_{2}$ is evolved. ${ }^{22}$ More recently, a Keggin-type POM was immobilized in the cavities of HKUST-1 together with Au nanoparticles and the authors suggested that the POMs act as electrons and protons reservoirs to boost $\mathrm{CO}_{2}$ photoreduction into $\mathrm{CO}$ and $\mathrm{CH}_{4}{ }^{23}$ In the present work we aim at combining the two approaches previously developed by our groups for targeting a single (POM,cat)@MOF composite, namely (PW $\left.12, \mathrm{Cp}^{*} \mathrm{Rh}\right) @ \mathrm{UiO}-67$, to explore the potential impact of the co-immobilization of polyoxometalates and $\mathrm{Cp} * \mathrm{Rh}$ catalytic complexes on the photocatalytic performances for $\mathrm{CO}_{2}$ conversion. Note that while the encapsulation of $\mathrm{PW}_{12}$ and the $\mathrm{RhH}(\mathrm{CO})\left(\mathrm{PPh}_{3}\right)_{3}$ complex in MIL-101(Cr) for hydroformylation of 1-octene was previously reported, ${ }^{24}$ the release of the $\mathrm{Rh}$ complex along the catalytic process was observed for this composite system. Herein, we consider an alternative approach where the catalytic complex is immobilized in the framework, acting as a constitutive MOF linker.

In view of the level of structural complexity reached in the (PW $\mathrm{PW}_{12}, \mathrm{Cp} \mathrm{Rh}^{\mathrm{N}}$ @UiO-67 solid, we use here, besides standard characterizations, an in-depth combination of Density Functional Theory (DFT) calculations and Pair Distribution Function (PDF) analysis using laboratory X-rays. To the best of our knowledge, such an investigation of POM@MOF and (POM,cat)@MOF composites by PDF has not been reported so far. Also, the mutual interactions between the MOF host and the POM and catalyst guests were probed using ${ }^{1} \mathrm{H},{ }^{13} \mathrm{C}$, and ${ }^{13} \mathrm{P}$ solid state NMR, in line with the computational chemistry 
findings. We finally report a significant enhancement of the photocatalytic performances of ( $\left.\mathrm{PW}_{12}, \mathrm{Cp} * \mathrm{Rh}\right) @ U$ UiO-67 for the conversion of $\mathrm{CO}_{2}$ into formate and hydrogen when compared to its POM-free counterpart.

\section{RESULTS AND DISCUSSION}

Synthesis and Characterizations. The ( $\left.\mathrm{PW}_{12}, \mathrm{Cp} * \mathrm{Rh}\right) @ U i \mathrm{U}-67$ compound was synthesized in two steps combining reported procedures for the synthesis of PW $12 @ U i 0-67^{12}$ and Cp*Rh@UiO-678 (Figure 1a). UiO-67 is built from the connection of $\mathrm{Zr}_{6}$ clusters by biphenyl-4,4'-dicarboxylate (bpdc) linkers. First, the encapsulation of the POMs in the octahedral cavities of Ui0-67 was achieved by in situ synthesis in DMF at $120^{\circ} \mathrm{C}$. The resulting PW ${ }_{12} @ U i 0-67$ composite was then reacted with the molecular complex $\mathrm{Cp} * \mathrm{Rh}$ (bpydc) $\mathrm{Cl}_{2}$ (bpydc $=2,2^{\prime}$-bipyridine-5,5'-dicarboxylic acid) in water at room temperature for $24 \mathrm{~h}$, following a post-synthetic linker exchange procedure. The percentage of the bpdc linkers exchanged in the MOF as well as the amount of POMs and Rh immobilized in the cavities were evaluated by EDS and elemental analyses, leading to

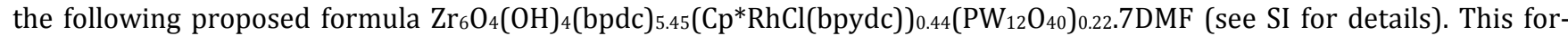
mula indicates the presence of an average of one POM and two Cp*Rh complexes every 5 unit-cells i.e. every 5 cavities. The molar Rh complex loading (7\%) is close to the $10 \%$ optimal loading determined in our previous study. ${ }^{8}$ For comparison purposes in the present work, the $\mathrm{Cp} * \mathrm{Rh} @ U \mathrm{UiO}-67$ solid with the same percentage of $\mathrm{Cp} * \mathrm{Rh}$ complex (7\%) was also synthesized.

The powder X-ray diffraction measurements (Figure S1) of the (PW $\left.\mathrm{PW}_{12}, \mathrm{Cp} * \mathrm{Rh}\right) @ U i \mathrm{U}-67$ composite, compared to that of UiO67, and PW12@UiO-67, confirm that the crystallinity of the MOF host is maintained upon the in situ incorporation of the POM and of the $\mathrm{Cp} * \mathrm{Rh}$ complex introduced post-synthetically. For the PW $\mathrm{PW}_{12} @ \mathrm{UiO}-67$ and (PW $\left.12, \mathrm{Cp} * \mathrm{Rh}\right) @ U i 0-67$ composites, we could unambiguously assign the additional broad signal visible at low $(2 \theta)$ angles under the Bragg peaks to the diffuse scattering signal of the encapsulated $\left[\mathrm{PW}_{12} \mathrm{O}_{40}\right]^{3-}$ in the MOF cavities, the PDF analysis excluding the possible presence of a separated amorphous phase (see below). Infrared (IR) spectroscopy (Figure S2) indicate both the presence of the POM, the UiO$67 \mathrm{MOF}$ and the $\mathrm{Cp} * \mathrm{Rh}$ complex, with $\mathrm{P}-\mathrm{O}$ vibrations around $1100 \mathrm{~cm}^{-1}, \mathrm{~W}=0$ vibrations around $980 \mathrm{~cm}^{-1}$, W-O vibrations around $780 \mathrm{~cm}^{-1}$ and carboxylate vibrations between 1350 and $1650 \mathrm{~cm}^{-1}$. Note that the IR spectrum of (PW $\left.\mathrm{PW}_{12}, \mathrm{Cp} * \mathrm{Rh}\right) @ \mathrm{UiO}-$ 67 does not unambiguously show the presence of the POM as the characteristic bands of the polyanion are in the same frequency domain than bands of the rhodium functionalized MOF host. However, Raman spectra evidence the presence of the $\mathrm{PW}_{12}$ and $\mathrm{Cp} * \mathrm{Rh}$ species in the MOF (Figure S3). The vibrational bands of the POM are visible in the $960-1040 \mathrm{~cm}^{-1} \mathrm{region}$ and typical Raman features of the $\mathrm{Cp} * \mathrm{Rh}$ complex are clearly observed in the (PW $\left.{ }_{12}, \mathrm{Cp} * \mathrm{Rh}\right) @ U i 0-67$ at $440(\delta(\mathrm{N}-\mathrm{Rh}-\mathrm{N}))$, 1324 and $1506 \mathrm{~cm}^{-1}(v(\mathrm{C}=\mathrm{N})+v(\mathrm{C}=\mathrm{C}))$. Combined with scanning electron microscopy (SEM) images (Figure 2a), energy dispersive X-ray spectroscopy (EDS) mapping (Figures 2b-d) suggests a uniform distribution of the POM and of the Rh complex within these particles. As expected, the Brunauer-Emmett-Teller (BET) surface area (Figure S4) calculated from the $\mathrm{N}_{2}$ isotherm significantly decreases from UiO-67 to $\mathrm{PW}_{12} @ U$ UiO-67 and (PW $\left.\mathrm{PW}_{12}, \mathrm{Cp} * \mathrm{Rh}\right) @ U$ UiO-67 as a result of the successive incorporation of the POM and of the Rh complex, with $2300 \mathrm{~m}^{2} \mathrm{~g}^{-1}, 1034 \mathrm{~m}^{2} \mathrm{~g}^{-1}$ and $930 \mathrm{~m}^{2} \mathrm{~g}^{-1}$, respectively. The transformed UV-visible absorption spectrum of ( $\left.\mathrm{PW}_{12}, \mathrm{Cp}^{*} \mathrm{Rh}\right) @ U i 0-67$ compared to that of $\mathrm{Cp}^{*} \mathrm{Rh} @ U \mathrm{UiO}-67$ exhibits a slight red-shift of the Rh-localized d-d transition ${ }^{8}$ (Figure S5). This suggests a direct interaction between the POM and the Cp*Rh catalyst.

The ${ }^{1} \mathrm{H}$ and ${ }^{13} \mathrm{C}$ MAS NMR spectra (Figures S6A, and S6B) of Cp*Rh@UiO-67 and (PW ${ }_{12}, \mathrm{Cp} * \mathrm{Rh}$ )@UiO-67, when compared to those of $\mathrm{Cp} * \mathrm{Rh}$, UiO-67, and PW $12 @ U i O-67$, confirm the incorporation of the Cp*Rh complex into the UiO-67's framework.

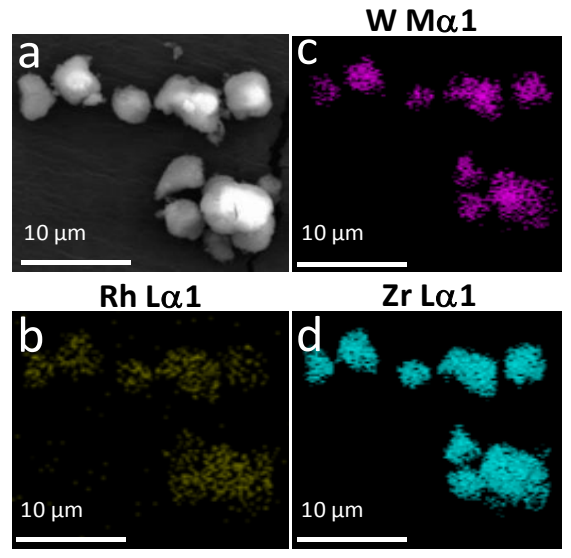

Figure 2. : (a) SEM image of ( $\left.\mathrm{PW}_{12}, \mathrm{Cp} * \mathrm{Rh}\right) @ U i \mathrm{U}-67$, (b), (c), (d) EDS mapping of Rh, W, Zr respectively. 


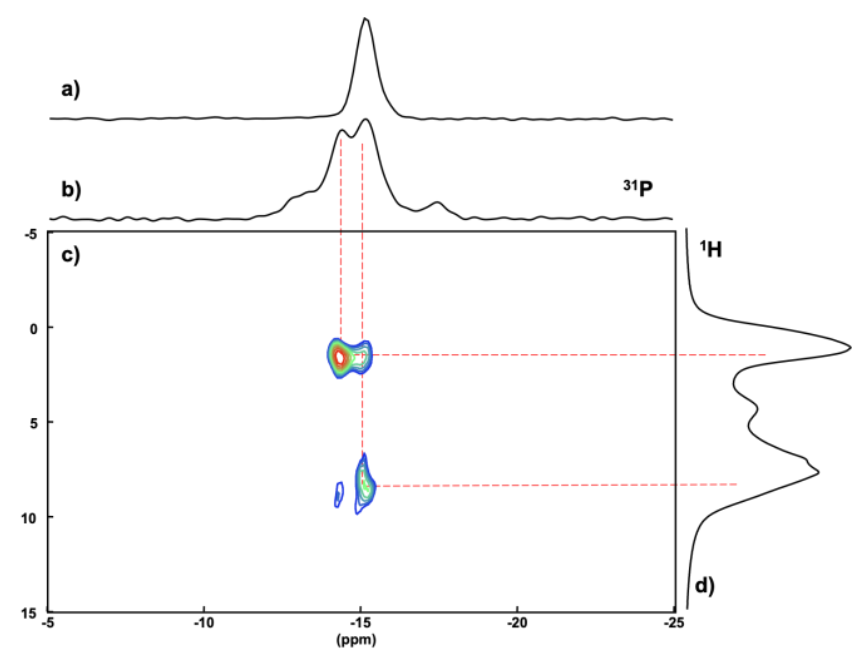

Figure 3. ${ }^{31} \mathrm{P}\left\{{ }^{1} \mathrm{H}\right\}$ CPMAS NMR spectra of (a) PW ${ }_{12} @ U i O-67$ and (b) $(\mathrm{PW} 12, \mathrm{Cp} * \mathrm{Rh}) @ U i 0-67$, and $(\mathrm{c}){ }^{31} \mathrm{P}\left\{{ }^{1} \mathrm{H}\right\} \mathrm{HETCOR}$ and $(\mathrm{d}){ }^{1} \mathrm{H}$ MAS NMR spectra of (PW $12, \mathrm{Cp} * \mathrm{Rh}) @ U$ UiO-67.

The ${ }^{1} \mathrm{H}$ NMR spectra of UiO-67 and PW $12 @ U i O-67$ exhibit the expected resonances of aromatic linker's protons (7.1 and 7.9 ppm) and of hydroxyl groups of the inorganic node (0.2-3.7 ppm) including those of defect sites. ${ }^{12,25}$ New resonances at 1.1 1.2 ppm observed in Cp*Rh@UiO-67 and (PW $\left.{ }_{12}, \mathrm{Cp}^{*} \mathrm{Rh}\right) @ U i 0-67$ solids correspond to methyl protons of the Cp* moiety, however significantly shielded with respect to the resonance at $1.9 \mathrm{ppm}$ observed in the spectrum of the $\mathrm{Cp} * \mathrm{Rh}_{(\mathrm{bpydc}) \mathrm{Cl}}$ precursor. Furthermore, the absence of the signal at $12.5 \mathrm{ppm}$ due to the acid protons of the carboxylic groups of the precursor indicates the full condensation of the (bpydc) linker with the inorganic node after the ligand exchange process. ${ }^{13} \mathrm{C}$ NMR also confirms the inclusion of both the Cp* complex and bpydc ligand with the appearance of resonances at ca. 9 and $98 \mathrm{ppm}$, and ca. $154 \mathrm{ppm}$, respectively. It should be mentioned that the excessive line broadening observed in both ${ }^{1} \mathrm{H}$ and ${ }^{13} \mathrm{C}$ spectra of $\left(\mathrm{PW}_{12}, \mathrm{Cp} \mathrm{R}^{*} \mathrm{Rh}\right) @ U \mathrm{UiO}-67$ when compared to those of PW $\mathrm{PW}_{12} @ U$ UiO-67 or Cp*Rh@UiO-67 should indicate major disorder within the structure when the POM and the $\mathrm{Cp}^{*}$ complex are introduced together.

The ${ }^{31} \mathrm{P}$ MAS NMR spectra of PW $12 @ U i 0-67$ and $\left(\mathrm{PW}_{12}, \mathrm{Cp} \mathrm{P}^{*} \mathrm{Rh}\right) @ U \mathrm{UiO}-67$ are shown in Figures 3a and 3b, respectively. A single signal is observed at -15.1 ppm for PW P. $_{12}$ UiO-67 reflecting an homogeneous environment of the POM in UiO-67, while two main resonances at -15.2 and -14.4 ppm can be distinguished in the case of $\left(\mathrm{PW}_{12, \mathrm{Cp}} * \mathrm{Rh}\right) @ U$ UiO-67. This latter result suggests that the POMs occupy two distinct structural positions within the pores of Cp*Rh@UiO-67. Further evidences of that are provided by 2D NMR ${ }^{31} \mathrm{P}-{ }^{1} \mathrm{H}$ HETCOR experiments (Figure 3c). The spectrum shows that the ${ }^{31} \mathrm{P}$ signal at $-15.2 \mathrm{ppm}$ correlates preferably with the ${ }^{1} \mathrm{H}$ signal of aromatic protons at $8.3 \mathrm{ppm}$, while the second ${ }^{31} \mathrm{P}$ signal at -14.4 ppm correlates with the $\mathrm{Cp}^{*}$ moiety peak at $1.6 \mathrm{ppm}$. This clearly indicates that two POM environments co-exist within the (PW $\left.{ }_{12}, \mathrm{Cp} \mathrm{p}^{*} \mathrm{Rh}\right) @ U \mathrm{UiO}-67$ material, i.e. one closer to the Cp* fragment (-14.4 ppm) and another closer to the organic linker ($15.2 \mathrm{ppm}$ ). Quantitatively, they are distributed almost equally (47:53 for -14.4 and -15.2 ppm respectively).

DFT calculations. In a first step, simulated annealing (SA) and dispersion-corrected DFT-D3 geometry optimizations were performed in order to identify the most likely position of the POM in UiO-67 and qualify its host-guest interactions with the hybrid framework (see SI for details). SA simulations provided recurrent successful insertion of PW 12 in the octahedral cage of UiO-67. Figures $4 \mathrm{a}$ and S7 illustrates its position and interactions in the solid as obtained from the DFT-D3 level geometry optimization of the most stable conformation extracted from SA. The POM is located at the center of the cavity and interacts equally with the twelve bpdc linkers of the octahedral cage. It is stabilized through a network of $\mathrm{O}_{(\mathrm{PW} 12) \cdots} \mathrm{H}_{(\mathrm{bpdc})}$ hydrogen bonds between its terminal oxygen atoms and two aromatic hydrogen atoms of each bpdc linker within 2.4-2.7 $\AA$ distances. Overall, the host-guest interaction energy, which emanates from both electrostatic interactions and multiple hydrogen bonds, is estimated from DFT calculations around $200 \mathrm{kcal}^{\mathrm{mol}}{ }^{-1}$. This high affinity of the MOF for the POM results from the confinement of the POM in the rather small size pore of UiO-67.

We then investigated the positioning of the Rh catalytic complex in the PW12@UiO-67 solid considering the replacement of one bpdc linker per octahedral cage by one $\mathrm{Cp} \mathrm{p}^{*} \mathrm{Rh}(\mathrm{bpydc})$ catalytic linker (Figures 4 and S7). Two situations were distinguished whereby the $\mathrm{Cp} * \mathrm{Rh}$ catalytic moiety is oriented either towards an empty, i.e. POM-free, neighboring cage (Figure $4 \mathrm{~b}$, position "out") or towards the same cage than the PW 12 guest (Figure 4c, position "in"). The DFT-D3 level geometry optimizations of the "out" position shows a similar $\{\mathrm{POM}, \mathrm{MOF}\}$ interaction energy than in the catalyst-free MOF. By contrast the comparison of the "out" and "in" models shows that interactions between the POM and the Cp* Rh component of the catalyt-

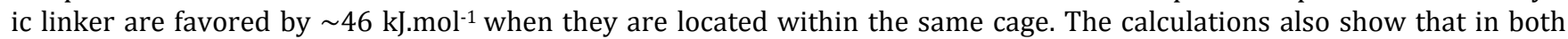
"out" and "in" positions short-range interactions of the PW 12 with the linkers of the MOF are maintained through a series of hydrogen-bond type interactions within the 2.3-3 A range of distances.

The DFT calculations are fully consistent with the NMR experimental observations. The severe line broadening of the NMR signals reflects the structural disorder in the (POM,cat)@MOF material as the result of a loss of local symmetry when both 
the Keggin-POM and the Rh-catalyst are introduced into the MOF. Notably, the incorporation of either the POM or the Rhcatalyst induces much less disorder within the resulting POM@MOF or Cat@MOF solids.

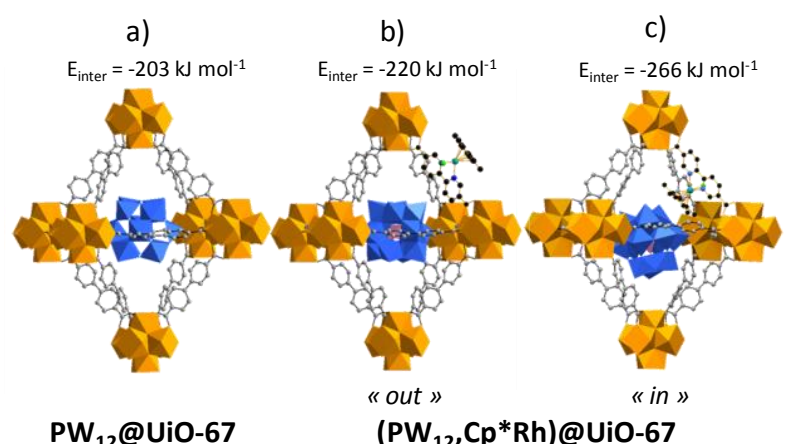

Figure 4. Details of $\mathrm{PW}_{12} @ U \mathrm{UiO}-67$ and (PW $\left.12, \mathrm{Cp} * \mathrm{Rh}\right) @ U i 0-67$ models. (a) $\mathrm{PW}_{12}$ in $\mathrm{PW}_{12} @ \mathrm{UiO} \mathrm{O}-67$ and (b)-(c) in (PW $12, \mathrm{Cp} * \mathrm{Rh}) @ U i 0-67$ as obtained from SA and DFT-D3 level geometry optimizations. The POM and the Rh-catalytic center are modeled within different cages ("out" position, b) or within the same cage ("in" position, c). Interaction energies between PW 12 and the MOF host are given for each model. $\mathrm{WO}_{6}$, blue polyhedra; $\mathrm{ZrO}_{8}$, orange polyhedra; $\mathrm{Rh}$, dark green sphere; 0 , red spheres; $\mathrm{C}$, grey spheres; N, dark blue spheres; $\mathrm{Cl}$, green spheres. $\mathrm{H}$ atoms are omitted.

Along this line, DFT calculations show that the POM is perfectly centered in the cage of the PW12@UiO-67 solid (Figure 4a), i.e. in absence of the Rh-catalyst, with thus only little effect on long and short range orders. By contrast, the presence of the Rh-catalyst displaces the POM off the center of the cage when the Cp* moiety points toward the PW 12 POM (Figure 4c), which results in a new POM environment characterized by a ${ }^{31} \mathrm{P}$ NMR signal at $-14.4 \mathrm{ppm}$ as revealed by the $2 \mathrm{D}{ }^{13} \mathrm{P}-{ }^{1} \mathrm{H}$ correlation experiment. The ${ }^{31} \mathrm{P}$ NMR signal at $-15.2 \mathrm{ppm}$ is assigned to the POM centered in the cage with no close interaction with the $\mathrm{Cp}^{*}$ of the $\mathrm{Rh}$-complex. This situation occurs when no Rh-catalyst is present within the cage or when the $\mathrm{Cp}^{*}$ ligand is oriented toward an adjacent neighboring cage (Figure 4b). Finally the experimental NMR data confirm the occurrence of the two distinct DFT local structures of the POM distributed equally.

\section{Pair Distribution Functions analysis.}

The catalytic properties of the (POM,cat)@MOF solid rely on the integrity of its (POM, cat, MOF) components and on subtle interactions between the immobilized (POM, cat) species and the MOF host. Rietveld refinement methods from powder Xray diffraction (PXRD) patterns, based on the Bragg peaks analysis arising from symmetry in polycrystalline solids, may be used to locate guest species while retrieving the host's crystal structure. However, such techniques - even using high resolution synchrotron data - are not suitable when the immobilized guests exhibit a strong positional disorder in the porous host. Although many POM@MOF composites have been reported for catalysis purposes, ${ }^{26,27}$ there has been no report so far on POM@MOF crystal structure determination from powders, besides the proposed positioning of PW 12 in NU-1000 by Farha et al. from difference electron density maps. ${ }^{28,29}$ PDF data collection, which includes both Bragg and diffuse scattering, was thus selected as a valuable technique to overcome the above limitations.

The PDF signal reflects the probability of finding a pair of atoms at a distance $r$ in the structure and may be described as a one-dimensional function that exhibits peaks at r-values corresponding to characteristic inter atomic distances within the sample. The strength of this method lies in the quantitative structural information provided about short, middle and longrange orders, including all the deviations from the average structure. ${ }^{30}$ In the present POM@MOF and (POM,cat)@MOF materials, the contributions arising from both the host and the guests are expected to induce a strong peak overlap making the interpretation of the PDF data quite challenging. To overcome this limitation, an elegant approach was proposed by Chapman et al., namely the differential PDF (d-PDF) method, ${ }^{31}$ whereby the local structure of the guest, the host-guest interactions and the structural modifications of the host are retrieved through the subtraction of the PDF of the guest-free host (as a reference) from that of the guest-loaded PDF. In this context, Chapman et al. studied $\mathrm{N}_{2}$ adsorbed in Prussian blue whereby the d-PDF refinement showed a slight expansion of the host network and a confinement of the loaded $\mathrm{N}_{2}$ molecules close to the pore centers. ${ }^{30}$ In another challenging case, Platero-Prats et al. showed the formation of Cu-oxo clusters within the small pores of NU-1000 during the atomic layer deposition of $\mathrm{Cu}^{32}$ The further refinement of the d-PDF highlighted the formation under reducing atmosphere of metallic $\mathrm{Cu}^{0}$ nanoparticles of $4 \mathrm{~nm}$ and $<1 \mathrm{~nm}$ sizes within the pores, followed by their transformation into cuprite $\mathrm{Cu}_{2} \mathrm{O}$ nanoparticles under oxidative atmosphere. However, the use of PDF and d-PDF methods is still limited in the field of guest@MOF materials, with only a few reports, most of which concerning the adsorption of small molecules. ${ }^{30,23-35}$ To the best of our knowledge, the investigation of POM@MOF and (POM,cat)@MOF composites by differential PDF analysis is the first of the kind.

In a first step, PDF analysis was performed on PW $12 @ U i 0-67$ using UiO-67 as a reference, which will act as a proof-ofconcept for the applicability of the d-PDF method to our composites. The experimental PDF profile of the UiO-67 (Figure S8) is characterized by a series of narrow peaks on the selected $r$-range (1-40 $\mathrm{A})$ which typically attest the high crystallinity of the sample, i.e. with short, middle and long range orders. All intense peaks were assigned in a similar fashion than previously done on the related UiO-66 material, ${ }^{36}$ reflecting the reported crystal structure of UiO-67. ${ }^{37}$ More details may be found in 
SI. Turning to the PW12@Ui0-67 composite, its experimental PDF profile contains all the expected features of the UiO-67 host while presenting particularly intense additional peaks below 10.0 Å (Figure 5a).
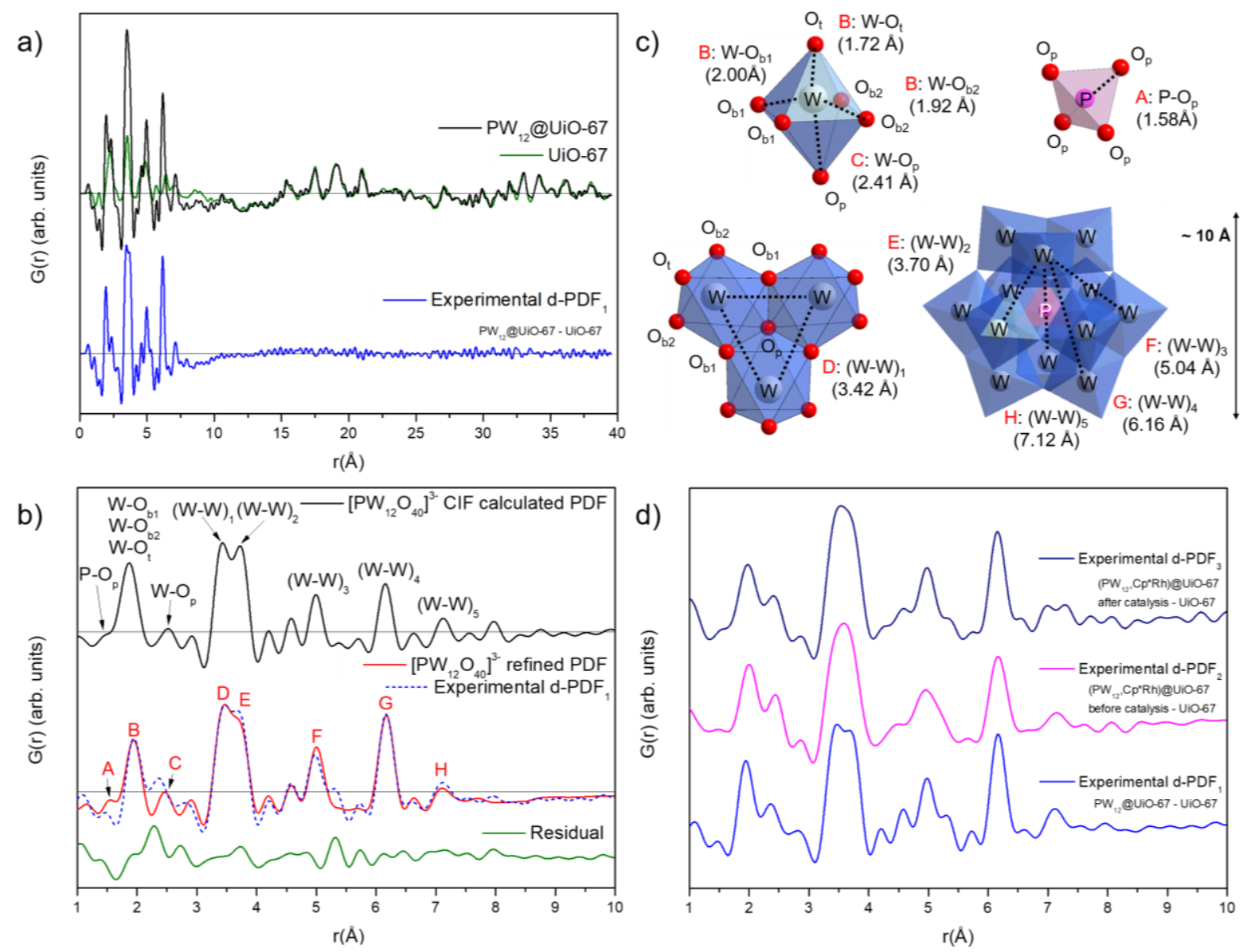

Figure 5. (a) Experimental PDF data for PW $12 @ U i 0-67$ (black) and the guest-free UiO-67 (green) and the corresponding d-PDF for PW 12 encapsulated in UiO-67 (blue) determined by subtracting the PDF of UiO-67 from the PDF of PW $12 @ U i 0-67$. (b) Comparison of the calculated PDF of an isolated PW 12 (black) and the experimental d-PDF of PW 12 in UiO-67 (blue dotted line), superimposed with the refined d-PDF (red) fitted using the $\left[\mathrm{PW}_{12} \mathrm{O}_{40}\right]^{3-}$ structural model from CIF file (JCPDS 00-050-0304) ${ }^{34}$ and residual profile (green). A-H labels of peaks correspond to the indicated refined distances in the $\mathrm{POM}$ components as illustrated in (c) for the $\mathrm{PO}_{4}$ tetrahedron, the $\mathrm{WO}_{6}$ octahedron, the trimer of $\mathrm{WO}_{6}$ octahedra, and within the full $\mathrm{PW}_{12}$ polyoxometalate structure. $\mathrm{WO}_{6}$, blue octahedra, $\mathrm{PO}_{4}$, pink tetrahedron, 0 , red spheres, $\mathrm{W}$, grey spheres, $\mathrm{P}$ pink sphere. (d) Experimental d-PDFs (numbered 1 to 3 ) for PW12 in PW12@UiO-67 (blue), in (PW12,Cp*Rh)@Ui0-67 before catalysis (magenta) and in (PW12,Cp*Rh)@UiO-67 after catalysis (navy blue) determined by subtracting the PDF of UiO-67 from the PDF of PW $12 @ U i O-67,\left(P W_{12}, \mathrm{Cp}^{*} \mathrm{Rh}\right.$ )@UiO-67 before and after catalysis, respectively.

Knowing the respective scattering factors of the POM and MOF atoms $\left(f_{W}>>f_{Z r}>>f_{P}>f_{0}>f_{C}\right.$ ), these narrow and intense peaks clearly emanate from the heavy $\mathrm{W}$ scatterers and indicate their short-range structural organization in line with the presence of the encapsulated POM.

Importantly, the two experimental PDF profiles perfectly overlap at long range order (15.0 to $40.0 \AA$ A), confirming the similar high crystallinity of the UiO-67's framework upon the encapsulation of $\mathrm{PW}_{12}$ by in situ synthesis, as already observed from their PXRD patterns (Figure S1). This long range feature allowed the calculation of the differential PDF in optimal conditions. The resulting d-PDF (Figure 5a) is dominated by intense and narrow peaks in the low $r$ region with a strong dampening above $10.0 \AA$.

The d-PDF was compared with the calculated PDF of an isolated $\mathrm{PW}_{12}$ extracted from reported crystallographic data (JCPDS 00-50-0304 CIF file) ${ }^{38}$ over the 1-10 Å r-range (Figure 5b). The two profiles match remarkably well whereby all peaks of the experimental d-PDF are equally well described (shape and position) in the calculated PDF of PW12, allowing an exhaustive assignment of its characteristic interatomic distances. The first short distance peak at $1.5 \AA$ (A) corresponds to the P-O bond length of the central $\mathrm{PO}_{4}$ tetrahedron of the POM. The second and third peaks (B, C) at 2.0 and $2.4 \AA$, respectively, correspond to the length of various $\mathrm{W}-\mathrm{O}$ bonds constituting the $\mathrm{WO}_{6}$ octahedra.

The intense peaks (D-G) at 3.5, 3.7, 5.0, 6.2 and 7.1 $\AA$, are assigned to various $\mathrm{W}$-W distances inside the $\mathrm{PW}_{12}$ structure. In addition, a very good agreement is found over the 1-8 ̊̊r-range between the observed d-PDF and the theoretical one calcu- 
lated from the DFT models of UiO-67 and PW $12 @ U i 0-67$ (Figure S9a). As detailed in SI, the disagreement between the experimental and theoretical d-PDF profiles at long range ( $>8 \AA$ ) emanates from the absence of POM's ordering in the PW12@Ui0-67 composite, by contrast with the virtual ordering induced by the DFT periodic models.

Overall, the structural integrity of the PW 12 upon encapsulation in UiO-67 is clearly demonstrated. We further attempted a refinement of the POM's atomic positions whereby it is constrained to the space group Pn-3m (Figure 5b, Table S3). The relatively good quality of the refinement $\left(\mathrm{R}_{\mathrm{w}} \sim 28.2 \%\right)$ and the corresponding structural model (Figure $5 \mathrm{c}$ ) indicate that the original structure of the POM is indeed fully preserved upon its immobilization in UiO-67. The residual difference between experimental and calculated d-PDFs might be assigned to errors induced by the PDF subtraction step at low r-values, and/or potential positional atomic disorder and symmetry loss within the POM structure induced by the proximity of UiO67's ligands and nodes.

In a second step, the d-PDF related to the $\left(\mathrm{PW}_{12}, \mathrm{Cp} * \mathrm{Rh}\right) @ \mathrm{UiO}-67$ catalyst was investigated using UiO-67 as a reference. The PDF of the $\left(\mathrm{PW}_{12}, \mathrm{Cp} * \mathrm{Rh}\right) @$ UiO-67 exhibits all expected features of UiO-67 showing that the linker exchange did not induce major changes in the structure or important loss of crystallinity. The perfect overlap of ( $\left.\mathrm{PW}_{12}, \mathrm{Cp} * \mathrm{Rh}\right) @ \mathrm{UiO}-67$ 's and UiO-67's PDF profiles at large r-values (15-40 ̊) allowed us to calculate the related d-PDF (Figure S10) reflecting the POM's structure. Consistently, this d-PDF (Figure 5d) is very similar to the d-PDF obtained for the POM in PW $12 @ U i O-67$, i.e. prior to the linker exchange (Figure 5d), confirming that the post-synthetic linker exchange has no impact on the structural integrity of PW 12 . It is worth mentioning here that further attempts to identify the contribution of the catalytic $\mathrm{Cp} * \mathrm{Rh}$ ligand in the d-PDFs were unsuccessful due to the low amount of $\mathrm{Rh}$ in the sample and also to the coincidence between $\mathrm{Rh}-\mathrm{Cl}$ distances and those of $\mathrm{W}-\mathrm{O}$ distances characteristic of the POM. Finally, the collection of PDF data of the (PW $\left.{ }_{12}, \mathrm{Cp} * \mathrm{Rh}\right) @ U i 0-67$ composite after photocatalysis (see below) showed no modification of the d-PDF profile associated to the POM (Figure S11), demonstrating that it remains intact upon the photocatalytic step (Figure 5d).

Overall, the PDF analyses provide an unequivocal signature of the immobilized PW 12 in UiO-67. The d-PDF directly reflects its local structure through the inventory of interatomic distances within the PW 12 moiety, from which a structural refinement was performed (Table S3). The similarity of the experimental d-PDFs associated to PW 12 before and after catalysis provides a direct proof of the integrity of the POM after photocatalysis.

Photocatalytic Activities. Considering that $\mathrm{Cp} * \mathrm{Rh} @ U$ UiO-67 was active for the $\mathrm{CO}_{2}$ reduction under visible-light irradiation, 8 the photocatalytic activity of the ( $\left.\mathrm{PW}_{12}, \mathrm{Cp} * \mathrm{Rh}\right) @ U \mathrm{UiO}-67$ composite was investigated. The production of $\mathrm{H}_{2}$ and formate were thus compared for the Cp*Rh@UiO-67 and (PW $12, \mathrm{Cp} * \mathrm{Rh}$ )@UiO-67 materials (Figures 6 and S12-S13), using acetonitrile as the solvent and TEOA as electron and proton donor and with $\mathrm{Ru}(\mathrm{bpy})_{3} \mathrm{Cl}_{2}$ as an external photosensitizer. No other reaction products were detected neither with $\mathrm{Cp} * \mathrm{Rh} @ U i 0-67$ nor $\left(\mathrm{PW}_{12}, \mathrm{Cp} * \mathrm{Rh}\right) @ U i 0-67$. The $\mathrm{H}_{2}$ production performed by the (Cp*Rh,PW 12 @Ui0-67 catalyst increases progressively before reaching a plateau after $3 \mathrm{~h}$, in a similar fashion than observed with Cp*Rh@UiO-67 (Figure 6a). Remarkably, we estimate at $3 \mathrm{~h}$ that the $\mathrm{H}_{2}$ production is enhanced by a factor of 2.5 in the (Cp*Rh,PW 12 )@UiO-67 composite with respect to the POM-free Cp*Rh@UiO-67. The production of formate follows the same trend, being similarly enhanced (Figure 6b). Thus, the presence of the POM in the Cat@MOF photosystem enhances both the hydrogen and formate production. The addition of a fresh solution of $\mathrm{Ru}(\mathrm{bpy}){ }_{3} \mathrm{Cl}_{2}$ after $2 \mathrm{~h}$ of reaction results in a new cycle of $\mathrm{H}_{2}$ production with similar initial TOF $\left(\sim 0.30 \mathrm{~min}^{-1}\right.$, Figure S14), clearly showing that the system is mainly limited by the consumption of the photosensitizer. Control experiments with no irradiation did not show any formate production nor hydrogen production. A very minor $\mathrm{H}_{2}$ production can be observed with the Rh-catalyst-free PW12@Ui0-67 (Figure 6a). By comparison with the experiment in absence of composite, this production can be fully assigned to some catalytic activity of $\mathrm{Ru}(\mathrm{bpy})_{3} \mathrm{Cl}_{2}$ (Figure 6a). Importantly, no significant differences are detected in the XRD (Figure S1), IR (Figure S2) and EDS-SEM analyses (Figure S15, Table S1) between the composite before and after the reaction, confirming its stability upon photocatalysis in line with the above PDF findings.

The above results raise the question of the role of the $\mathrm{PW}_{12}$ in the photocatalytic reduction of $\mathrm{CO}_{2}$ by the (PW12,Cp*Rh)@UiO-67 composite. While the great majority of molecular $\mathrm{CO}_{2}$ reduction catalysts convert $\mathrm{CO}_{2}$ into $\mathrm{CO}$, polypyridyl complexes of rhodium are part of the very few reported catalysts for the reduction of $\mathrm{CO}_{2}$ to formate. ${ }^{39,40}$ Recently DFT computations elucidated the catalytic mechanism involved in the photoreduction of $\mathrm{CO}_{2}$ into formate and $\mathrm{H}_{2}$ by a series of $\left[\mathrm{Rh}(\mathrm{R}-\mathrm{bpy}) \mathrm{Cp}^{*} \mathrm{Cl}\right]^{+}$catalysts and identified the formation of the $\mathrm{Rh}-\mathrm{H}$ hydride as the key step for both reactions. ${ }^{41}$ Once the Rh-catalyst is immobilized in the UiO-67's framework as a constitutive linker, its catalytic activity towards the reduction of $\mathrm{CO}_{2}$ into formate and $\mathrm{H}_{2}$ upon illumination is maintained ${ }^{8}$ whereby an oxidative quenching mechanism prevails in the presence of the TEOA sacrificial electron donor as recently reported. ${ }^{7}$

Considering the (PW $12, \mathrm{Cp} * \mathrm{Rh}) @ U i 0-67$ material, DFT calculations also tend to show that the electronic structure of the Rh-catalyst is little affected by $\mathrm{PW}_{12}$ (Figure S16). These calculations indeed do not reveal any significant change in both the distribution and the absolute levels of the HOMO and LUMO of the Rh-complex in presence of the PW 12 moiety, meaning that no overlap is observed between the frontier molecular orbitals of the POM and of the Rh-catalyst. Moreover, as expected due to the oxidant behaviour of the PW 12 moiety, ${ }^{42}$ the LUMO of the POM is placed, in the absolute scale, at more negative energies than the LUMO of the Rh-complex, thus preventing it from acting as an electron relay. Building on these findings and on the absence of significant catalytic activity of the Rh-free $\mathrm{PW}_{12} @ \mathrm{UiO}-67$ solid towards $\mathrm{CO}_{2}$ reduction (see Figure 6), we likely consider $\mathrm{PW}_{12}$ in the $\left(\mathrm{PW}_{12}, \mathrm{Cp} * \mathrm{Rh}\right) @ U \mathrm{UiO}-67$ material as assisting the catalytic $\mathrm{CO}_{2}$ reduction as a proton relay (Figure S17), knowing the outstanding proton conductivity of POMs. ${ }^{43}$ This role is here supported by the direct evidence of 
the high mobility of acidic protons in the anhydrous $\mathrm{PW}_{12}{ }^{44}$ together with reports of remarkably enhanced proton conductivities in $\mathrm{PW}_{12} @ \mathrm{MOF} / \mathrm{COFs}$ materials. ${ }^{45-48}$

As MOFs are obtained mostly as polycrystalline powders, depositing them on transparent conducting supports allows developing easy-to-use and recyclable setups. Having this in mind, the stability and recyclability of the (PW $\left.12, \mathrm{Cp}^{*} \mathrm{Rh}\right) @ \mathrm{UiO} \mathrm{-67}$ composite upon photocatalysis were further assessed using thin films rather than suspensions, by drop-casting (DC) (PW $12, \mathrm{Cp} * \mathrm{Rh}) @ U i O-67$ on an indium tin oxide (ITO) plate. Only a slight decrease of the formate production is observed after the first run (8\%) and the second run of irradiation (9\%) (Figure S18). Furthermore it can be noticed that much higher formate TONs are achieved with DC-thin films than with the suspension (175 compared to 14.6, at $3 \mathrm{~h}$ ). This can be attributed to the better illumination of the crystallites deposited on the ITO plate when compared to usual photocatalytic experiments performed on a suspension, as we recently observed for the $\mathrm{P}_{2} \mathrm{~W}_{18} \mathrm{Co}_{4} @ \mathrm{MOF}-545$ photosystem. ${ }^{49}$ While all particles deposited on ITO are indeed exposed to light, the less efficient exposure of crystallites to light when in suspension (random orientation of crystallites, light diffusion by the suspension...) may be responsible for the lower TONs observed.
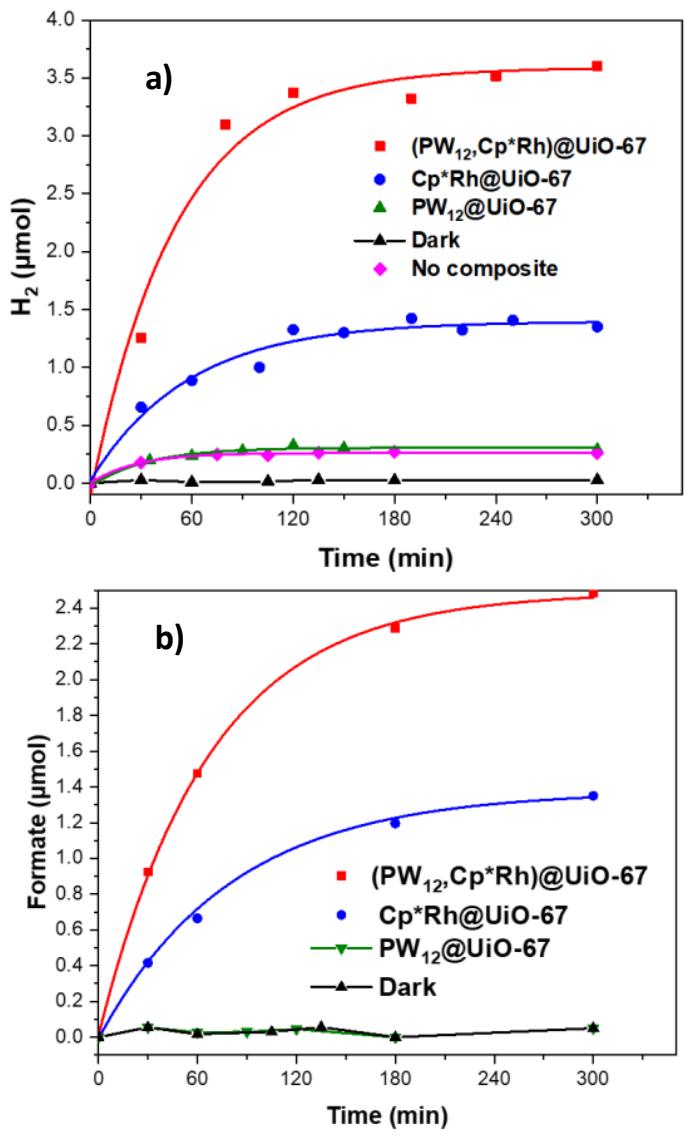

Figure 6. Kinetics of (a) $\mathrm{H}_{2}$ and (b) $\mathrm{HCOO}^{-}$production during $\mathrm{CO}_{2}$ photoreduction reaction by (PW $\left.12, \mathrm{Cp} * \mathrm{Rh}\right) @ \mathrm{UiO}-67$ compared to Cp*Rh@UiO-67. Reactions conditions: $0.17 \mu \mathrm{mol}$ of catalyst, $0.8 \mathrm{~mL}$ of $\mathrm{CH}_{3} \mathrm{CN} / \mathrm{TEOA} 5: 1,1 \mathrm{mM} \mathrm{Ru}(\mathrm{bpy})_{3} \mathrm{Cl}_{2}, 280 \mathrm{~W}, \lambda>415 \mathrm{~nm}$.

\section{CONCLUSIONS}

We successfully co-immobilized a POM and a Cp*Rh catalyst in the (PW $\left.\mathrm{PW}_{12}, \mathrm{Cp} * \mathrm{Rh}\right) @ U$ UiO-67 materials and showed that the reduction of $\mathrm{CO}_{2}$ into formate and the production of $\mathrm{H}_{2}$ are significantly enhanced when compared to those observed with the POM-free Cp*Rh@UiO-67 material while the Cp*Rh-free PW ${ }_{12} @ U i O-67$ is not catalytically active. Catalytic studies were performed on crystallites in suspensions. The ( $\left.\mathrm{PW}_{12}, \mathrm{Cp} * \mathrm{Rh}\right) @ U i 0-67$ composite was also deposited as films on IT0 plates, providing evidence for the stability and the recyclability of the system as well as improved performances compared to suspensions. Importantly, differential Pair Distribution Function analysis was used for the first time to characterize a POM@MOF composite and allowed to prove unambiguously the integrity of the POM before and after catalysis. Also, solidstate NMR nicely confirmed the two environments of the POMs within the MOF's cavities determined by DFT calculations, with the $\mathrm{Cp} * \mathrm{Rh}$ moiety pointing either towards a POM-free cavity or towards a POM. Such POM-Cp*Rh interactions might explain the beneficial role of the POMs in the photocatalytic reduction of $\mathrm{CO}_{2}$. DFT calculations also suggest that the electronic structure of the Rh-catalyst is hardly affected by PW12 and that the LUMO of the POM is placed, in the absolute scale, at more negative energies than the LUMO of the Rh-complex thus preventing it from acting as an electron relay. We thus rather propose that the POM could play the role of a proton relay. Further investigations on these hypotheses will be explored in future works. In particular we plan to immobilize Keggin ions possessing different acidities and redox potentials 
such as $\left[\mathrm{SiW}_{12} \mathrm{O}_{40}\right]^{4-},\left[\mathrm{BW}_{12} \mathrm{O}_{40}\right]^{5-}$, $\left[\mathrm{PTi}_{2} \mathrm{~W}_{10} \mathrm{O}_{40}\right]^{7-}$ and $\left[\mathrm{PMo}_{12} \mathrm{O}_{40}\right]^{3-}$. Overall, our findings open perspectives on the investigation of novel POM-based MOFs for enhanced photocatalytic performances.

\section{EXPERIMENTAL SECTION}

$\mathrm{H}_{3} \mathrm{PW}_{12} \mathrm{O}_{40}, 50 \mathrm{UiO}-67,12,37 \mathrm{PW}_{12} @ U i \mathrm{U}-67,{ }^{12} \mathrm{Cp} * \mathrm{Rh}(\mathrm{bpydc}) \mathrm{Cl}_{2}{ }^{8}$ were synthesized according to reported procedures. Details for the synthesis of $\mathrm{Cp} * \mathrm{Rh} @ U i \mathrm{O}-67$ are given in SI. All of the other reagents were purchased from commercial sources and used as received.

Synthesis of (PW $\mathbf{1 2}, \mathbf{C p} * \mathbf{R h}) @ U i 0-67$. In a 10 mL glass flask, PW $12 @ U i O-67$ (50 mg, 1.87 10 $0^{-5} \mathrm{~mol}$ ) and Cp*Rh(bpydc) $\mathrm{Cl}_{2}$ (17 mg, $310^{-5} \mathrm{~mol}$ ) were suspended in $5 \mathrm{~mL}$ degassed deionized water. This solution was stirred at room temperature for $24 \mathrm{~h}$. The solid was isolated by centrifugation and suspended in $5 \mathrm{~mL}$ of DMF. After standing at room temperature for $1 \mathrm{~h}$ the suspension was centrifuged and the solvent was decanted. The obtained yellow solid (40 $\mathrm{mg}$ ) was then washed with ethanol and finally dried under reduced pressure at room temperature.

Physical Methods. Infrared (IR) spectra were recorded on a Nicolet 30 ATR 6700 FT spectrometer. Energy dispersive spectroscopy (EDS) measurements were performed on a JEOL JSM 5800LV apparatus. $\mathrm{N}_{2}$ adsorption isotherms were obtained at $77 \mathrm{~K}$ using a BELsorp Mini (Bel, Japan). Prior to the analysis, approximately $50 \mathrm{mg}$ of sample were evacuated at $120^{\circ} \mathrm{C}$ under primary vacuum over $5 \mathrm{~h}$. Elemental analyses were performed by CREALINS, 5 Rue de la Doua, 69100 Villeurbanne, France (heavy elements, ICP-AES analyses), and by the Service de Microanalyse of CNRS, ICSN, 91198 Gif-sur-Yvette, France (C, H, N). UV-vis spectra were recorded on a Perkin Elmer Lambda 750 UV/Vis/NIR spectrometer. ${ }^{1} \mathrm{H},{ }^{13} \mathrm{C}$ and ${ }^{31} \mathrm{P}$ MAS NMR spectra were recorded on a Bruker AVANCE-500 spectrometer (Larmor frequencies of 500.162, 125.774 and $202.465 \mathrm{MHz}$, respectively) at $292 \mathrm{~K}$ using a $2.5 \mathrm{~mm}$ MAS probe. The following conditions were used for recording the ${ }^{1} \mathrm{H}$ MAS NMR spectra: the length of $90^{\circ}{ }^{1} \mathrm{H}$ pulse was $2.1 \mu \mathrm{s}$, and the delay time between scans was $9.5 \mathrm{~s}$, which satisfied $5 \times \mathrm{T}_{1}$ condition. 8 scans were collected for each 1D ${ }^{1} \mathrm{H}$ MAS NMR spectrum. ${ }^{31} \mathrm{P}$ MAS NMR spectra with high power proton decoupling were recorded with or without cross-polarization (CP) denoted below as ${ }^{31} \mathrm{P}$ CPMAS NMR and ${ }^{31} \mathrm{P}$ MAS NMR. The following conditions were used for recording the spectra with $\mathrm{CP}$ both in $1 \mathrm{D}$ and in $2 \mathrm{D}{ }^{1} \mathrm{H}-{ }^{31} \mathrm{P}$ heteronuclear correlation (HETCOR) NMR experiments: the proton radiofrequency (rf) field was $75 \mathrm{kHz}$, contact time was $10 \mathrm{~ms}$ at the Hartmann-Hahn matching condition of $64 \mathrm{kHz}$. The single pulse excitation ${ }^{31} \mathrm{P}$ MAS NMR spectra were recorded with $90^{\circ}$ flip angle pulses of $2.3 \mu \mathrm{s}$ duration and $10 \mathrm{~s}$ recycle delay. In these experiments a high power proton decoupling of $50 \mathrm{kHz} \mathrm{rf}$ field was used only during the acquisition time. A 1024 scans were collected for each 1D ${ }^{31} \mathrm{P}$ CPMAS NMR and ${ }^{31} \mathrm{P}$ MAS NMR spectrum. For 2D CPMAS ${ }^{1} \mathrm{H}-{ }^{31} \mathrm{P}$ HETCOR NMR experiments a total of $16 \mathrm{t}_{1}$ increments with 1024 scans each were collected. The experimental parameters for the ${ }^{13} \mathrm{C}$ CPMAS NMR experiments were $1 \mathrm{~s}$ delay time between scans and $2 \mathrm{~ms}$ contact time. The spinning rate was $10 \mathrm{kHz}$ for ${ }^{31} \mathrm{P}$ and ${ }^{13} \mathrm{C}$ NMR experiments, while for ${ }^{1}{ }^{1} \mathrm{H}$ MAS NMR experiments $30 \mathrm{kHz}$ spin rate was employed. ${ }^{1} \mathrm{H}$ and ${ }^{13} \mathrm{C}$ chemical shifts were referenced with respect to TMS, whereas ${ }^{31} \mathrm{P}$ chemical shifts to $85 \%$ $\mathrm{H}_{3} \mathrm{PO}_{4}$, as external standards, respectively, with accuracy of $\pm 0.5 \mathrm{ppm}$. Raman spectra were measured on a home-made Raman spectrometer at $532 \mathrm{~nm}$, the spectrum being acquired with a fiber coupled spectrometer coupled to a CCD camera (IsoPlane SCT 320 mounted with a PIXIS, using a 1200 lines grating; Princeton Inst.). An interferential filter was used to cut the elastic scattering. The spectral resolution was set to $8 \mathrm{~cm}^{-1}$. The laser power was adjusted to about $1 \mathrm{~mW}$ on the sample to avoid laser damage.

DFT calculations. In a first step, the position and orientation of the guest POM was sampled during a simulated annealing procedure, allowing to visit the void volume of UiO-67 (i.e. the octahedral cages). Non-bonded interaction energies between the POM and the hybrid UiO-67 framework were described with the cvff forcefield, ${ }^{51}$ in which van der Waals (Lennard-Jones potentials) and electrostatic interactions were explicitly included. The atomic charges for the POM and the UiO-67 were calculated by the charge-equilibration method. In a second step, low energy PW $W_{12} @ U i 0-67$ candidates were extracted from the SA results and further evaluated at the dispersion-corrected DFT-D3 level calculations with the Vienna Ab-initio Simulation Package VASP. ${ }^{52,53}$ The ( $\left.\mathrm{PW}_{12}, \mathrm{Cp}^{*} \mathrm{Rh} @ U \mathrm{UiO}-67\right)$ solid was constructed replacing one pbdc linker with a $\mathrm{Cp}^{*} \mathrm{Rh}(\mathrm{bpydc})$ linker oriented either towards the POM or towards an empty cage, for comparison purposes. A planewave basis set with the projector augmented wave (PAW) scalar-relativistic pseudopotentials ${ }^{54}$ was employed for all geometry and electronic calculations. The electron-ion interactions were described by the PAW method in the implementation of Kresse and Joubert. ${ }^{55}$ Geometry optimizations were performed with the Perdew-Burke-Ernzerhof (PBE) exchange-correlation functional. ${ }^{56}$ The long-range weak dispersion interactions were taken into account using the semi empirical VdW method of Grimme DFTD3. ${ }^{57} \mathrm{~A}$ plane-wave cutoff of $400 \mathrm{eV}$, for the construction of the electronic wave functions, was found to be suitable for convergence of the system. The integration over the irreductible Brillouin zone was carried out using the gamma point. Atomic positions were optimized until the forces on all atoms were smaller than $0.02 \mathrm{eV} \AA^{-1}$. Once the PW $12 @ U i 0-67$ and (PW $\mathrm{PW}_{12}, \mathrm{Cp} * \mathrm{Rh}$ )@UiO-67 periodic models were fully optimized, the interaction energy (or binding energy) between the POM and the UiO-67 or Cp*Rh@UiO-67 hosts was then estimated through single-point calculations.

PDF. X-ray total scattering data were measured with a Bruker D8 ADVANCE diffractometer equipped with a Göbel mirror and a LYNXEYE detector, with Mo K $\alpha$ radiation $($ mean $\lambda(\mathrm{K} \alpha 1 \alpha 2)=0.71073 \AA$ ) at room temperature. These data were used for both X-Ray Diffraction (XRD) pattern and Pair Distribution Function (PDF) analysis. The sample preparation consists first in a supercritical $\mathrm{CO}_{2}$ washing/activation step of the samples in order to remove all the solvent molecules inside the MOF pores after the synthesis. Then, inside a glovebox under Ar atmosphere, a few tens of milligrams of powder are placed in a thin-walled $(0.01 \mathrm{~mm})$ borosilicate glass capillary of $1.0 \mathrm{~mm}$ diameter. A higher $\mathrm{Cp} * \mathrm{Rh}$ loading $(\sim 20 \%)$ than for the 
photocatalytic experiments was here used for $\mathrm{Cp} * \mathrm{Rh} @ U i 0-67$ and $\left(\mathrm{PW}_{12}, \mathrm{Cp} * \mathrm{Rh}\right) @ U$ UiO-67 in order to maximize the signal of these complexes. The ( $\left.\mathrm{PW}_{12}, \mathrm{Cp} * \mathrm{Rh}\right) @ U i 0-67$ sample after photocatalysis was prepared in similar conditions than described in the "Photocatalytic Activity Measurements" section below. In order to obtain a sufficient amount of powder, three batches were prepared with the quantities of both solvent and catalyst multiplied by three (4.2 mg of (PW $\left.\left.{ }_{12}, \mathrm{Cp} * \mathrm{Rh}\right) @ U i 0-67\right)$. After an irradiation time of $90 \mathrm{~min}$, the suspensions were centrifuged and the solvent was decanted. The obtained powders were then washed two times with isopropanol and dried at room temperature, then activated under vacuum at $120^{\circ} \mathrm{C}$ for a night prior PDF data collection. Measurements were performed from $Q_{\min }=0.12 \AA^{-1}$ to $Q_{\max }=17.0 \AA^{-1}(Q=4 \pi \sin \theta / \lambda)$ on rotating capillaries with evolving counting parameters as a function of Q-range in order to optimize the counting rate at high $Q$. The final XRD diagram was thus obtained from the combination of 7 patterns, converted in counts per second, with the following parameters $2 \theta_{\mathrm{i}}\left({ }^{\circ}\right)-2 \theta_{\mathrm{f}}\left({ }^{\circ}\right)$-step size $\left(^{\circ}\right)$-step time(s): 0.8-31-0.02-2, 29-61-0.04-6, 59-91-0.06-15, 89-121-0.1-40 (twice) and 119-150-0.1-100 (twice) for a total measuring time of 34 hours. Additional scattering measurements from empty capillary were performed in the same conditions for background subtraction. Raw data were treated using the PDFgetX3 program $^{58}$ to obtain the total PDF G(r) function.

d-PDF calculation and refinement. The experimental differential PDFs (d-PDFs) were obtained by subtraction of the UiO67 PDF from that of the PW $\mathrm{PW}_{12} @ U \mathrm{UiO}-67$ sample and the (PW $\left.\mathrm{PW}_{12}, \mathrm{Cp} * \mathrm{Rh}\right) @ U \mathrm{UiO}-67$ sample.

The d-PDF was refined with a $\left[\mathrm{PW}_{12} \mathrm{O}_{40}\right]^{3-}$ structural model using the DIFFEV software included in the DISCUS suite package. 59,60 The initial $\left[\mathrm{PW}_{12} \mathrm{O}_{40}\right]^{3-}$ structural model was built from the crystalline structure of $\mathrm{H}_{3} \mathrm{PW}_{12} \mathrm{O}_{40} .6 \mathrm{H}_{2} \mathrm{O}$ with the space group Pn-3m (n ${ }^{\circ} 224$ ) (cubic) (JCPDS 00-050-0304) ${ }^{37}$ removing the structure water molecules and the 3 protons which compensate the POM negative charge, using the DISCUS software. The refinement was performed according to the procedure described by Neder and Proffen. ${ }^{58,59}$ The following parameters were refined considering the POM symmetry: the scale factor, the atomic positions (other than particular positions), the isotropic atomic displacement parameters ( $B_{\text {iso }}$ ) and the dynamic correlation factor (delta2). The interval in which the atomic position of the terminal oxygen $\mathrm{O}_{\mathrm{t}}$ can vary has been constrained to maintain a distance W- $\mathrm{O}_{\mathrm{t}}$ consistent with a double bond $\mathrm{W}=\mathrm{O}$ (around $1.7 \AA$ A). Metallic silicon (Si) served as a standard material to determine the experimental resolution effect factor $Q_{\text {damp }}$ (refined $Q_{\text {damp }}=0.028 \AA^{-1}$ ).

PDF calculation from DFT models. The PDF calculations from UiO-67 and PW12@Ui0-67 DFT models were performed using the PDFgui software ${ }^{61}$ with periodic boundary conditions. For each atom types, small isotropic atomic displacements (U $=0.001 \AA$ ) were considered in the calculation to obtain a PDF peak broadening consistent with experiment.

Photocatalytic Activity Measurements. Photochemical Reactions were performed using a $280 \mathrm{~W}$, high pressure Xe arc lamp (Newport Instruments). The beam was passed through a water infrared filter, a collimating lens and a filter holder equipped with a $415 \mathrm{~nm}$ band pass filter (Asahi Spectra). Samples were prepared in a $1 \mathrm{~cm}$ path length quartz sealed cuvette (Hellma) which was placed in a temperature-controlled cuvette holder (Quantum Northwest) maintained at $20{ }^{\circ} \mathrm{C}$ with a circulated water bath. A $\mathrm{CH}_{3} \mathrm{CN}$ /TEOA mixture 5:1 was used as a solvent $\left(\mathrm{CH}_{3} \mathrm{CN}\right.$ and TEOA purchased from Sigma Aldrich and used without further purification). The photosensitizer utilized was a $1.0 \mathrm{mM}$ solution of $\mathrm{Ru}(\mathrm{bpy}){ }_{3} \mathrm{Cl}_{2}(\mathrm{pur}-$ chased from Sigma Aldrich used without further purification). $1.4 \mathrm{mg}$ of (PW $\left.{ }_{12}, \mathrm{Cp} * \mathrm{Rh}\right) @ U i O-67$ and $0.9 \mathrm{mg}$ of Cp*Rh@UiO67, which corresponds to $0.17 \mu \mathrm{mol}$ of $\mathrm{Cp} * \mathrm{Rh}$ catalyst, were used for the photocatalytic studies. Samples were saturated with $\mathrm{CO}_{2}$ via directly bubbling $\mathrm{CO}_{2}$ through the solution mixture for $20 \mathrm{~min}$. During irradiation, the samples were vigorously stirred and aliquots of gas and liquid were analyzed. $\mathrm{H}_{2}$ measurements (aliquots of $50 \mu \mathrm{L}$ of the headspace) were performed by gas chromatography on a Shimadzu GC-2014 equipped with a Quadrex column, a Thermal Conductivity Detector and using $\mathrm{N}_{2}$ as a carrier gas. Formate concentration was determined using a Metrohm 883 Basic IC plus ionic exchange chromatography instrument, using a Metrosep A Supp 5 column and a conductivity detector. A typical measurement requires the sampling of $200 \mu \mathrm{L}$ of solution, followed by a 100 dilution in deionised $18 \mathrm{M} \Omega$ water and injection of $20 \mu \mathrm{L}$ into the IC chromatograph. All TONs and TOFs were estimated by normalizing the production of $\mathrm{H}_{2}$ and HCOO- with the number of nmol of $\mathrm{Cp} * \mathrm{Rh}$ catalyst.

Drop-casting thin films (DC-films) preparation. We showed in our previous study on $\mathrm{P}_{2} \mathrm{~W}_{18} \mathrm{Co}_{4} @ \mathrm{MOF}-545^{50}$ that the catalytic activity of a thin film is related to the proportion of efficiently illuminated crystallites and thus depends on its thickness. A film prepared by drop-casting (DC) offers the largest proportion of Cat@MOF crystallites exposed to light due to its lower thickness when compared to a film obtained by an electrophoretic method. In the present study, thin films were thus prepared using the DC method in order to optimize the catalytic activity. A suspension of $1 \mathrm{mg}$ of (PW $\left.\mathrm{PW}_{12}, \mathrm{Cp} * \mathrm{Rh}\right) @ \mathrm{UiO}$ $67 \mathrm{in} 1 \mathrm{~mL}$ of EtOH was sonicated for $15 \mathrm{~min} .10 \mu \mathrm{L}$ of this suspension was deposited on an ITO plate (6 mm x $6 \mathrm{~mm}$ ) and dried in air.

\section{ASSOCIATED CONTENT}

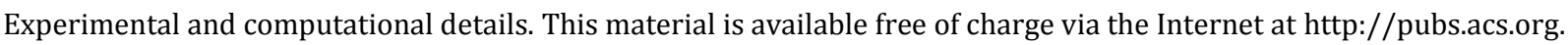

\section{AUTHOR INFORMATION}

\section{Corresponding Author}

* anne.dolbecq@uvsq.fr

* capucine.sassoye@upmc.fr

* caroline.mellot-draznieks@college-de-france.fr 


\section{Author Contributions}

"A. L. and Y. B. contributed equally.

\section{Funding Sources}

This work was supported by CNRS, UVSQ, the Ministère de l'Enseignement supérieur, de la Recherche et de l'Innovation, the French National Research Agency (ANR) as part of the "Investissements d'Avenir" program nANR-11-IDEX-0003-02 and CHARMMMAT ANR-11-LABX-0039. We also acknowledge financial support from the Paris Ile-de-France Region DIM "Respore". The calculations have been performed using the HPC resources from GENCI (CINES) through Grant 2016-097343.

\section{REFERENCES}

1 Evans, J. D.; Sumby, C. J.; Doonan, C. J. Post-synthetic metalation of metal-organic frameworks. Chem. Soc. Rev. 2014, $43,5933$.

2 Liang, Z.; Qu, C.; Xia, D.; Zou, R.; Xu, Q. Atomically Dispersed Metal Sites in MOF-based Materials for Electrocatalytic and Photocatalytic Energy Conversion. Angew. Chem. Int. Ed. 2018, 57, 9604-9633.

3 Luo, Y.-H.; Dong, L.-Z.; Liu, J.; Li, S.-L.; Lan, Y.-Q. From Molecular Metal Complex to Metal-Organic Framework: the $\mathrm{CO}_{2}$ Reduction Photocatalysts with Clear and Tunable Structure. Coord. Chem. Rev. 2019, 390, 86-126.

${ }^{4}$ Wang, C.; Xie, Z.; deKrafft, K. E.; Lin, W. Doping Metal-Organic Frameworks for Water Oxidation, Carbon Dioxide Reduction, and Organic Photocatalysis. J. Am. Chem. Soc. 2011, 133, 13445-13454.

${ }^{5}$ Choi, K. M.; Kim, D.; Rungtaweevoranit, B. ; Trickett, C. A.; Barmanbek, J. T. D.; Alshammari, A. S.; Yang, P.; Yaghi, O. M. Plasmon-Enhanced Photocatalytic $\mathrm{CO}_{2}$ Conversion within Metal-Organic Frameworks under Visible Light. J. Am. Chem. Soc. 2017, 139, 356-362.

${ }^{6}$ Kajiwara, T.; Fujii, M.; Tsujimoto, M.; Kobayashi, K.; Higuchi, M.; Tanaka, K.; Kitagawa, S. Photochemical Reduction of Low Concentrations of $\mathrm{CO}_{2}$ in a Porous Coordination Polymer with a Ruthenium(II)-CO Complex. Angew. Chem. Int. Ed. 2016, 55, 2697-2700.

7 Liao, W.-M.; Zhang, J.-H.; Wang, Z.; Yin, S.-Y.; Pan, M.; Wang, H.-P.; Su, C.-Y. Post-synthetic exchange (PSE) of UiO-67 frameworks with $\mathrm{Ru} / \mathrm{Rh}$ half-sandwich units for visible-light-driven $\mathrm{H}_{2}$ evolution and $\mathrm{CO}_{2}$ reduction. J. Mater Chem. A 2018, 6, 11337-1345.

${ }^{8}$ Chambers, M. B.; Wang, X.; Elgrishi, N.; Hendon, C. H.; Walsh, A.; Bonnefoy, J.; Canivet, J.; Quadrelli, E. A.; Farruseng, D.; Mellot-Draznieks, C.; Fontecave, M. Photocatalytic Carbon Dioxide Reduction with Rhodium-based Catalysts in Solution and Heterogenized within MetalOrganic Frameworks. ChemSusChem. 2015, 8, 603-608.

${ }_{9}^{9}$ Fei, H.; Sampson, M. D.; Lee, Y.; Kubiak, C. P.; Cohen, S. M. Photocatalytic $\mathrm{CO}_{2}$ Reduction to Formate Using a Mn(I) Molecular Catalyst in a Robust Metal-Organic Framework. Inorg. Chem. 2015, 54, 6821-6828.

${ }^{10}$ Lee, Y.; Kim, S.; Fei, H.; Kang, J. K.; Cohen, S. M. Photocatalytic $\mathrm{CO}_{2}$ reduction using visible light by metal-monocatecholato species in a metal-organic framework. Chem. Commun. 2015, 51, 16549-16552.

11 Wang, X.; Wisser, F. M.; Canivet, J.; Fontecave, M.; Mellot-Draznieks, C. Immobilization of a Full Photosystem in the Large-Pore MIL-101 Metal-Organic Framework for $\mathrm{CO}_{2}$ reduction. ChemSusChem. 2018, 11, 3315-3322.

12 Salomon, W.; Roch-Marchal, C.; Mialane, P.; Rouschmeyer, P.; Serre, C.; Haouas, M.; Taulelle, F.; Yang, S.; Ruhlmann, L.; Dolbecq, A. Immobilization of polyoxometalates in the Zr-based metal organic framework UiO-67. Chem. Commun. 2015, 51, 2972-2975.

${ }^{13}$ Wang, Sa-Sa; Yang, G.-Y. Recent Advances in Polyoxometalate-Catalyzed Reactions. Chem. Rev. 2015, 115, 4893-4962.

14 Salomon, W.; Paille, G.; Gomez-Mingot, M.; Mialane, P.; Marrot, J.; Roch-Marchal, C. ; Nocton, G. ; Mellot-Draznieks, C. ; Fontecave, M.; Dolbecq, A. Effect of Cations on the Structure and Electrocatalytic Response of Polyoxometalate-Based Coordination Polymers. Cryst. Growth Des. 2017, 17, 1600-1609.

${ }^{15}$ Cao, Y.; Chen, Q.; Shen, C.; He, L. Polyoxometalate-Based Catalysts for $\mathrm{CO}_{2}$ Conversion. Molecules 2019, $24,2069$.

16 Xie, S.-L.; Dong, L.-Z.; Li, S.-L.; Lan, Y.-Q.; Su, Z.-M. Hetero-metallic active sites coupled with strongly reductive polyoxometalate for selective photocatalytic $\mathrm{CO}_{2}$-to- $\mathrm{CH}_{4}$ conversion in water. Chem. Sci. 2019, 10, 185.

17. Lin, X.-X.; Liu, J.; Zhang, L.; Dong, L.-Z.; Xin, Z.-F.; Li, S.-L. ; Huang-Fu, X.-Q. ; Huang, K.; Lan, Y.-Q. Hydrophobic Polyoxometalate-Based Metal-Organic Framework for Efficient $\mathrm{CO}_{2}$ Photoconversion. ACS Appl. Mater. Interfaces 2019, 11, 25790.

${ }^{18}$ Huang, Q.; Liu, J.; Feng, L.; Wang, Q.; Guan, W.; Dong, L.-Z.; Zhang, L.; Yan, L.-K.; Lan, Y.-Q.; Zhou, H.-C. Multielectron transportation of polyoxometalate-grafted metalloporphyrin coordination frameworks for selective $\mathrm{CO}_{2}$-to- $\mathrm{CH}_{4}$ photoconversion. Natl.Sci. Rev. 2020, 7, 53.

${ }^{19}$ Ettedgui, J.; Diskin-Posner, Y.; Weiner, L.; Neumann, R. Photoreduction of Carbon Dioxide to Carbon Monoxide with Hydrogen Catalyzed by a Rhenium(I) Phenanthroline-Polyoxometalate Hybrid Complex. J Am. Chem. Soc. 2011, 133, 188-190.

${ }^{20} \mathrm{Ci}, \mathrm{C} . ;$ Carbo, J. J.; Neumann, R.; de Graaf, C.; Poblet, J.-M. Photoreduction Mechanism of $\mathrm{CO}_{2}$ to CO Catalyzed by a Rhenium(I)Polyoxometalate Hybrid Compound. ACS Catal. 2016, 6, 6422-6428.

${ }^{21}$ Haviv, E.; Shimon, L. J. W.; Neumann, R. Photochemical Reduction of $\mathrm{CO}_{2}$ with Visible Light Using a Polyoxometalate as Photoreductant. Chem. Eur. J. 2017, 23, 92-95

${ }^{22}$ Guo, W.; Lv, H.; Chen, Z.; Sullivan, K. P.; Lauinger, S. M.; Chi, Y.; Sumliner, J. M.; Lian, T.; Hill, C. L. Self-assembly of polyoxometalates, Pt nanoparticles and metal-organic frameworks into a hybrid material for synergistic hydrogen evolution. J. Mater. Chem. A 2016, 4, 59525957.

${ }^{23}$ Liu, S.-M.; Zhang, Z.; Li, X.; Jia, H.; Ren, M.; Liu, S. Ti-Substituted Keggin-Type Polyoxotungstate as Proton and Electron Reservoir Encaged into Metal-Organic Framework for Carbon Dioxide Photoreduction. Adv. Mat. Interfaces 2018, 5, 1801062.

${ }^{24}$ Sartipi, S.; Valero Romero, M. J.; Rozhko, E.; Que, Z.; Stil, H. A.; de With, J.; Kapteijn, F.; Gascon, J. Dynamic Release-Immobilization of a Homogeneous Rhodium Hydroformylation Catalyst by a Polyoxometalate Metal-Organic Framework Composite ChemCatChem 2015, 7, $3243-3247$

${ }^{25}$ Lawrence, M. C.; Schneider, C.; Katz, M. J. Determining the structural stability of UiO-67 with respect to time: a solid-state NMR investigation. Chem Commun. 2016, 52, 4971-4974.

${ }^{26}$ Samaniyan, M.; Mirzaei, M.; Khajavian, R.; Eshtiagh-Hosseini, H.; Streb. C. Heterogeneous Catalysis by Polyoxometalates in Metal-Organic Frameworks. ACS Catal. 2019, 9, 10174-10191.

27 Du, D.-Y.; Qin, J.-S.; Li, S.-L.; Su Z.-M.; Lan, Y.-Q. Recent advances in porous polyoxometalate based metal-organic framework materials. Chem. Soc. Rev. 2014, 43, 4615.

${ }^{28}$ Buru, C. T.; Li, P.; Mehdi, B. L.; Dohnalkova, A.; Platero-Prats, A. E.; Browning, N. D.; Chapman, K. W.; Hupp, J. T.; Farha. O. K. Adsorption of a Catalytically Accessible Polyoxometalate in a Mesoporous Channel-type Metal-Organic Framework. Chem. Mater. 2017, 29, 5174-5181. 
${ }^{29}$ Buru, C. T.; Platero-Prats, A. E.; Chica, M. G.; Kanatzidis, M. G.; Chapman, K. W.; Farha, O. K. Thermally induced migration of a polyoxometalate within a metal-organic framework and its catalytic effects. J. Mater. Chem. A, 2018, 6, 7389-7394.

${ }^{30}$ Egami, T.; Billinge, S. J. L. Underneath the Bragg Peaks: Structural Analysis of Complex Materials; Newnes, 2012.

31 Chapman, K. W.; Chupas, P. J.; Kepert, C. J. Selective Recovery of Dynamic Guest Structure in a Nanoporous Prussian Blue through in Situ X-Ray Diffraction: A Differential Pair Distribution Function Analysis. J. Am. Chem. Soc. 2005, 127, 11232-11233.

32 Platero-Prats, A. E.; Li, Z.; Gallington, L. C.; Peters, A. W.; Hupp, J. T.; Farha, O. K.; Chapman, K. W. Addressing the Characterisation Challenge to Understand Catalysis in MOFs: The Case of Nanoscale Cu Supported in NU-1000. Faraday Discuss. 2017, 201, 337-350.

${ }^{33}$ Sava Gallis, D. F.; Chapman, K. W.; Rodriguez, M. A.; Greathouse, J. A.; Parkes, M. V.; Nenoff, T. M. Selective O 2 Sorption at Ambient Temperatures via Node Distortions in Sc-MIL-100. Chem. Mater. 2016, 28, 3327-3336.

${ }^{34}$ Rangwani, S.; Howarth, A. J.; DeStefano, M. R.; Malliakas, C. D.; Platero-Prats, A. E.; Chapman, K. W.; Farha, O. K. Adsorptive Removal of $\mathrm{Sb}(\mathrm{V})$ from Water Using a Mesoporous Zr-Based Metal-Organic Framework. Polyhedron 2018, 151, 338-343.

35 Sava, D. F.; Rodriguez, M. A.; Chapman, K. W.; Chupas, P. J.; Greathouse, J. A.; Crozier, P. S.; Nenoff, T. M. Capture of Volatile Iodine, a Gaseous Fission Product, by Zeolitic Imidazolate Framework-8. J. Am. Chem. Soc. 2011, 133, 12398-12401.

${ }^{36}$ Bennett, T. D.; Todorova, T. K.; Baxter, E. F.; Reid, D.G.; Gervais, C.; Bueken, B.; Voorde, B. V.; de Vos, D. D.; A. Keen, D.; Mellot-Draznieks, C. Connecting Defects and Amorphization in UiO-66 and MIL-140 Metal-Organic Frameworks: A Combined Experimental and Computational Study. Phys. Chem. Chem. Phys. 2016, 18, 2192-2201.

37 Øien, S.; Wragg, D.; Reinsch, H.; Svelle, S.; Bordiga, S.; Lamberti, C.; Lillerud, K. P. Detailed Structure Analysis of Atomic Positions and Defects in Zirconium Metal-Organic Frameworks. Cryst. Growth Des. 2014, 14, 5370.

${ }^{38}$ Kremenovic A.; Spasojevic-de Bire A.; Dimitrijevic R.; Sciau P.; Mioc, U.B.; Colomban, Ph. Keggin's ion structural modification and expansion of dodecatungstophosphoric acid hexahydrate induced by temperature treatment: In situ X-ray powder diffraction and raman investigations, Solid State Ionics, 2000, 132, 39-53.

${ }^{39}$ Elgrishi, N.; Chambers, M. B.; Wang, X.; Fontecave, M. Molecular Polypyridine-Based Metal Complexes as Catalysts for the reduction of $\mathrm{CO}_{2}$. Chem. Soc. Rev. 2017, 46, 761-796.

${ }^{40}$ Takeda, H.; Cometto, C.; Ishitani, O.; Robert, M. Electrons,Photons, Protons and Earth-Abundant Metal Complexes for Molecular Catalysis of $\mathrm{CO}_{2}$ Reduction. ACS Catal. 2017, 7, 70-88.

${ }^{41}$ Todorova, T. K.; Huan, T. N.; Wang, X.; Agarwala, H.; Fontecave, M. Controlling Hydrogen Evolution during Photoreduction of $\mathrm{CO}_{2}$ to Formic Acid Using [Rh(R-bpy) $\left.\left(\mathrm{Cp}^{*}\right) \mathrm{Cl}\right]^{+}$Catalysts: A Structure-Activity Study Inorg. Chem. 2019, 58, 6893-6903.

${ }^{42}$ Lopez, X.; Bo, C.; Poblet, J. M. Electronic properties of polyoxometalates: electron and proton affinity of mixed-addenda Keggin and Wells-Dawson anions. J. Am. Chem. Soc. 2002, 124, 12574-12582.

${ }^{43}$ Zhai, L.; Haolong Li. H. Polyoxometalate-Polymer Hybrid Materials as Proton Exchange Membranes for Fuel Cell Applications Molecules 2019, 24, 3425-3445.

${ }^{44}$ Kolokolov, D. I.; Kazantsev, M. S.; Luzgin, M. V.; Jobic, H.; Stepanov. A. G. Direct ${ }^{2}$ H NMR Observation of the Proton Mobility of the Acidic Sites of Anhydrous 12-Tungstophosphoric Acid. ChemPhysChem. 2013, 14, 1783-1786.

${ }^{45}$ Liu, Y. W.; Yang, X.; Miao, J.; Tang, Q.; Liu, S. M.; Shi, Z.; Liu, S. X. Polyoxometalate-functionalized metal-organic frameworks with improved water retention and uniform proton-conducting pathways in three orthogonal directions. Chem. Commun. 2014, 50, 1002310026.

${ }^{46}$ Lai, X. Y.; Liu, Y. W.; Yang, G. C.; Liu, S. M. ; Shi, Z.; Lu, Y.; Luo, F.; Liu, S. X. Controllable proton-conducting pathways via situating polyoxometalates in targeting pores of a metal-organic framework. J. Mater. Chem. A 2017, 5, 9611-9617.

${ }^{47}$ Meng, X.; Wang, H.-N.; Song, S.-Y.; Zhang, H.-J. Proton-conducting crystalline porous materials. Chem. Soc. Rev. 2017, 46, 464-480.

${ }^{48}$ Ma, H. P.; Liu, B. L.; Li, B.; Zhang, L. M.; Li, Y. G.; Tan, H. Q.; Zang H. Y.; Zhu, G. S. Cationic Covalent Organic Frameworks: a Simple Platform of Anionic Exchange for Porosity Tuning and Proton Conduction. J. Am. Chem. Soc. 2016, 138, 5897-5903.

${ }^{49}$ Paille, G.; Gomez-Mingot, M.; Roch-Marchal, C.; Haouas, M.; Benseghir, Y.; Pino, T.; Ha-Thi, M.-H.; Landrot, G.; Mialane, P. ; Fontecave, M.; Dolbecq, A.; Mellot-Draznieks, C. Thin Films of Noble Metal-Free POM@MOF for Photocatalytic Water Oxidation. ACS Appl. Mater. Interfaces, 2019, 47837-47845.

50 Souchay, P. Ions Minéraux Condensés; Paris, 1969.

51 Maple, J.; Dinur, U.; Hagler, A. T. Derivation of force fields for molecular mechanics and dynamics from ab initio energy surfaces Proc. Nat. Acad. Sci. U.S.A.1988, 85, 5350.

52 Kresse, G.; Furthmüller, J. Efficiency of ab-initio total energy calculations for metals and semiconductors using a plane-wave basis set. Comput. Mater. Sci. 1996, 6, 15-50.

53 Kresse, G.; Furthmüller, J. Efficient iterative schemes for ab initio total-energy calculations using a plane-wave basis set. Phys. Rev. B 1996, 54, 11169-11186.

54 Blöchl, P. E. Projector augmented-wave method. Phys. Rev. B 1994, 50, 17953-17979.

55 Kresse, G.; Joubert, D. From ultrasoft pseudopotentials to the projector augmented-wave method. Phys. Rev. B 1999, 59, $1758-1775$.

56 Perdew, J. P.; Burke, K.; Ernzerhof, M. Generalized Gradient Approximation Made Simple. Phys. Rev. Lett. 1996, 77, $3865-3868$.

57 Grimme, S.; Antony, S.; Ehrlich, J.; Krieg, H. A consistent and accurate ab initio parametrization of density functional dispersion correction (DFT-D) for the 94 elements H-Pu. J. Chem. Phys. 2010, 132, 154104.

58 Juhás, P.; Davis, T.; Farrow, C. L.; Billinge, S. J. L. PDFgetX3: A Rapid and Highly Automatable Program for Processing Powder Diffraction Data into Total Scattering Pair Distribution Functions. J. Appl. Crystallogr. 2013, 46, 560-566.

${ }^{59}$ Neder, R. B.; Proffen, T. Diffuse Scattering and Defect Structure Simulations: A Cook Book Using the Program DISCUS; International Union of Crystallography Texts on Crystallography; Oxford University Press: Oxford, New York, 2008.

60 Proffen, T.; Neder, R. B. DISCUS, a Program for Diffuse Scattering and Defect Structure Simulations - Update. J. Appl. Crystallogr. 1999, $32,838-839$.

${ }^{61}$ L Farrow, C.; Juhas, P.; Liu, J. W.; Bryndin, D.; S Božin, E.; Bloch, J.; Proffen, T.; Billinge, S. PDFfit2 and PDFgui: Computer Programs for Studying Nanostructure in Crystals. J. Phys. Condens. Matter 2007, 19, 335219. 